\title{
Stability margin definition for a converter-grid system based on diagonal dominance property in the sequence-frame
}

\author{
Gabriele Amico, Agustí Egea-Àlvarez, Lie Xu \\ University of Strathclyde \\ 204 George Street, G1 1XW \\ Glasgow (UK) \\ gabriele.amico@strath.ac.uk
}

\author{
Paul Brogan \\ Siemens Gamesa Renewable Energy \\ Glasgow (UK)
}

\begin{abstract}
Keywords
$\ll$ Converter circuit $\gg, \ll$ Converter control $\gg, \ll$ Voltage Source Converter (VSC) 》, $\ll$ Power transmission $\gg, \ll$ Renewable energy systems $\gg$.
\end{abstract}

\begin{abstract}
The increased presence of power converters in power networks has set new power system stability challenges. New methodologies have been proposed to analyse these systems and one of the most promising is the impedance-based stability criterion. To date using this method the grid interfaced converter is represented in terms of impedances and it is assumed that the converter impedances within the sequence-frame are decoupled. However, coupling between the components of the sequence-frame converter impedances have been observed, and different studies have shown how relevant this might become to the stability performance. Hence, a stability margin that takes such coupling into account is presented in this paper. This results in a more conservative measure of the stability robustness of the system, compared to the gain and phase margin figures employed in the impedance-based stability criterion. The presented study has been used to assess how the relative stability of a VSC system connected to a weak grid varies with its operating point, and experimental data have been used to support the analytical study.
\end{abstract}

\section{Introduction}

Modern power networks have witnessed an increased presence of power electronic based equipment, such as in solar and wind power plants, or in HVDC links [1], [2]. The interconnection between power converter units and the grid has been seen to be responsible for various stability issues, such as cable due resonances [3] or sub-synchronous resonances in DFIG-based wind farms [4], or in weak grids [5], therefore challenging the preservation of grid stability [6].

The need to provide reliable solutions to such issues has driven the research for suitable methodologies and tools to model and analyse such converter-grid interface systems. Among the new methods (see for example [7]), the impedance-based stability criterion has been proposed in [8]. An advantage of this method is the possibility to simplify the stability study by representing the system in terms of impedances in the sequence-frame [9]. In doing so, the system typically is considered to be diagonal. Hence, by neglecting the usually weak coupling between the positive and negative sequence converter impedances, the Multiple-Input-Multiple-Output (MIMO) converter-grid system can be treated as two decoupled Single-Input-Single-Output (SISO) systems. By following this approach, SISO concepts like phase and gain margins come in handy, providing intuitive and easily interpretable indexes to quantify the relative stability of the system [10].

However, several studies have proved the existence of coupling between the positive and the negative sequence converter impedance terms [11], [12] discussing how this can become relevant to the stability assessment. The inclusion of such coupling in the relative stability study of the system requires 
the identification of MIMO stability margins, whose definitions are not as straightforward and intuitive as in the SISO scenario [10]. Different approaches have been presented in the control literature. The critical direction theory is proposed in [13], according to which at any frequency there is one specific vectorial direction of the applied perturbation which is relevant to the system stability. The structured singular-value method is proposed in [14], where a framework is built to generalise SISO stability margin definitions. Another attempt is the MIMO version of the circle theorem [10], whose effectiveness to assess the robustness of the system against different sources of uncertainty has been proved. A special case is represented by diagonal dominant systems [15], where more intuitive and graphically visualizable stability margin definitions are possible [10].

In this paper, some of the results presented in [16] on the stability study of diagonal dominant systems are applied to the power system context, to assess the stability performance of the inerconnection between a power converter unit and the grid. The approach proposed by the impedance-based stability criterion is taken, and the typically verified diagonal dominance property of the converter-grid system in the sequence-frame is used. A stability margin is presented, which is based on perturbation theory [17]. Compared to the gain and phase margin figures, which are used as a result of treating the system as two decoupled SISO sub-systems, i.e. ignoring the cross-coupling terms of the converter impedance, the proposed stability margin takes such coupling into account. A safer and more conservative evaluation of the system stability robustness can be obtained in this way. Moreover, negative values of the proposed stability margin, which are associated to scenarios where the diagonal dominance property of the system is not verified, are likely to indicate a less performing system, characterised by poorly damped dynamics. The paper is organised as follows. In section 2, a description of the methodology used to model a gridconverter system in the sequence-frame is presented, discussing how its diagonal dominance property is defined and how this is used to define the presented stability margin. Thereafter, in section 3, the study of the absolute and relative stability of a grid-connected Voltage Source Converter (VSC) prototype connected to a weak grid is conducted, comparing the SISO stability margins calculated according to the impedance-based stability criterion [8] with the proposed stability margin. Experimental results are also included to support the analytical study. Final conclusions are provided in section 4 .

\section{Study of the relative stability of a converter-grid interface in the sequence- frame: perturbation norm stability margin}

The presented stability margin is based on the property of diagonal dominance of the equivalent smallsignal model of a converter-grid system, in the sequence-frame. In section 2.1 the theory used to formulate such a model is described. Thereby, in section 2.2, the aforementioned stability margin is introduced. Finally, in section 2.3, a comparison between this stability margin and the SISO stability margins used in the application of the impedance-based stabiity criterion [8] is presented.

\subsection{Modelling of the converter-grid interface in the sequence-frame}

For the purpose of illustrating the modelling of a converter-grid interface in the sequence-frame, a prototype of such system, shown in Figure 1, is considered. This consists of a two-level Voltage Source Converter (VSC) connected to an RL grid. An RC filter is employed to attenuate the PWM harmonics generated by the converter. In the Figure, $i$ is the inverter output current, while $U$ is the voltage across the filter. Both of these signals are measured by the controller, together with the converter DC voltage, $U_{D C}$.

Taking the approach proposed in [8] to assess the stability of this system typology, its small-signal model is implemented in the sequence-frame. Such a model, which describes the linearised dynamics of the system at its operating point, is formulated as the circuit in Figure 2a. A Norton equivalent circuit is used to model the converter, while a Thévenin equivalent circuit is used to model the remaining parts of the electrical system. In Figure $2, Z_{\mathrm{c}}$ is the small-signal sequence-frame converter impedance, which also includes the reactor $L_{\mathrm{f}}$, whereas $Z_{\mathrm{Th}}$ is the small-signal Thévenin equivalent grid impedance, which includes the RC filter. It is noted that both $Z_{\mathrm{c}}$ and $Z_{\mathrm{Th}}$ are calculated in the sequence-frame. In the circuit, the signals $\Delta U$ and $\Delta i$ respectively represent the small-signal perturbations of $U$ and $i$ around their steady-state values $U_{0}, i_{0}$, taken at the system operation point. 


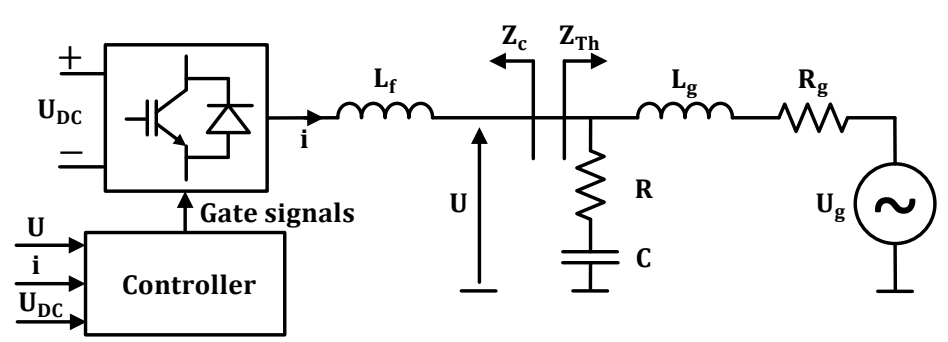

Fig. 1: One line diagram of the considered grid-connected VSC prototype.

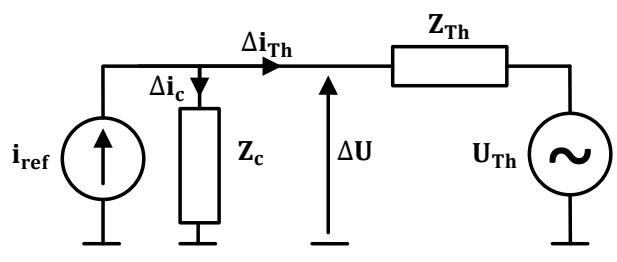

(a)

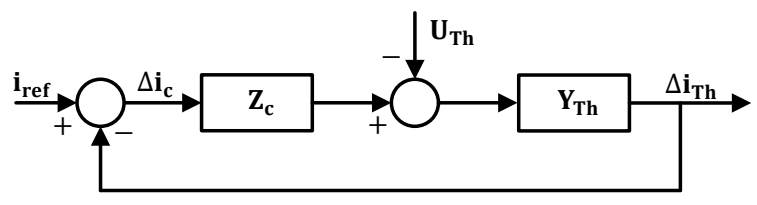

(b)

Fig. 2: (a) Electrical circuit equivalent to the small-signal model of the converter-grid system, linearised at its operating point. (b) Equivalent feedback loop.

Figure $2 \mathrm{~b}$ shows the equivalent feedback system of the circuit in Figure 2a. Its open-loop gain $L(s)$ is calculated as

$$
L(s)=Y_{\mathrm{Th}}(s) \cdot Z_{\mathrm{c}}(s)
$$

with $Y_{\mathrm{Th}}(s)=Z_{\mathrm{Th}}^{-1}(s)$. As it will be discussed in section 2.2, the presented definition of stability margin is based on the diagonal dominance property of the $I+\hat{L}(s)$ matrix over the Nyquist contour $\mathcal{D}$ [16], where $I$ is the $2 \times 2$ identity matrix and

$$
\hat{L}(s)=L^{-1}(s)=\left(Y_{\mathrm{Th}}(s) \cdot Z_{\mathrm{c}}(s)\right)^{-1}=Z_{\mathrm{c}}^{-1}(s) \cdot Y_{\mathrm{Th}}^{-1}(s)=Y_{\mathrm{c}}(s) \cdot Z_{\mathrm{Th}}(s)
$$

Hence, it is necessary to calculate the $\hat{L}(s)$ matrix, which in turns requires the calculation of both the small-signal converter admittance $Y_{\mathrm{c}}(s)=Z_{\mathrm{c}}^{-1}(s)$ and the small-signal impedance $Z_{\mathrm{Th}}(s)$. The former has been calculated based on the following equation [18]

$$
\begin{aligned}
& \Delta i_{\mathrm{p}}(s)=y_{\mathrm{c}, \mathrm{pp}}(s) \Delta U_{\mathrm{p}}(s)+y_{\mathrm{c}, \mathrm{pn}}(s) \Delta U_{\mathrm{n}}\left(s-2 j \omega_{0}\right) \\
& \Delta i_{\mathrm{n}}(s)=y_{\mathrm{c}, \mathrm{np}}(s) \Delta U_{\mathrm{p}}\left(s+2 j \omega_{0}\right)+y_{\mathrm{c}, \mathrm{nn}}(s) \Delta U_{\mathrm{n}}(s)
\end{aligned}
$$

where $\omega_{0}=2 \pi f_{0}$ (with $f_{0}$ representing the grid nominal frequency). $\Delta U_{\mathrm{pn}}(s)$ and $\Delta i_{\mathrm{pn}}(s)$ are the expression of $\Delta U(s)$ and $\Delta i(s)$ in the sequence-frame, respectively. The terms $y_{\mathrm{c}, \mathrm{pp}}(s), y_{\mathrm{c}, \mathrm{pn}}(s), y_{\mathrm{c}, \mathrm{np}}(s), y_{\mathrm{c}, \mathrm{nn}}(s)$ are the elements of $Y_{\mathrm{c}}(s)$. These have been calculated from the small-signal converter admittance in the $q d$-frame, and applying a methodology inspired by [9], [18], [12] to refer it to the sequence-frame. By shifting the second equation of (3) by $-2 \omega_{0}$, the following formulation is obtained for $Y_{\mathrm{c}}(s)$

$$
Y_{\mathrm{c}}(s)=\left[\begin{array}{cc}
y_{\mathrm{c}, \mathrm{pp}}(s) & y_{\mathrm{c}, \mathrm{pn}}(s) \\
y_{\mathrm{c}, \mathrm{np}}\left(s-2 j \omega_{0}\right) & y_{\mathrm{c}, \mathrm{nn}}\left(s-2 j \omega_{0}\right)
\end{array}\right]
$$

An equivalent formulation is obtained for the small-signal impedance matrix $Z_{\mathrm{Th}}(s)$. In particular, by assuming that the electrical impedances of the system are balanced, it results that $z_{\mathrm{Th}, \mathrm{pn}}(s)=z_{\mathrm{Th}, \mathrm{np}}(s)=0$ and $z_{\mathrm{Th}, \mathrm{pp}}(s)=z_{\mathrm{Th}, \mathrm{nn}}(s)=z_{\mathrm{Th}}(s)$, where $z_{\mathrm{Th}}(s)$ is the $a b c$-frame phase impedance [19]. Hence, the 
expression of $Z_{\mathrm{Th}}(s)$ is

$$
Z_{\mathrm{Th}}(s)=\left[\begin{array}{cc}
z_{\mathrm{Th}}(s) & 0 \\
0 & z_{\mathrm{Th}}\left(s-2 j \omega_{0}\right)
\end{array}\right]
$$

Finally, based on (2), the final expression of $\hat{L}(s)$ is

$$
\hat{L}(s)=\left[\begin{array}{cc}
y_{\mathrm{c}, \mathrm{pp}}(s) z_{\mathrm{Th}}(s) & y_{\mathrm{c}, \mathrm{pn}}(s) z_{\mathrm{Th}}\left(s-2 j \omega_{0}\right) \\
y_{\mathrm{C}, \mathrm{np}}\left(s-2 j \omega_{0}\right) z_{\mathrm{Th}}(s) & y_{\mathrm{c}, \mathrm{nn}}\left(s-2 j \omega_{0}\right) z_{\mathrm{Th}}\left(s-2 j \omega_{0}\right)
\end{array}\right]
$$

The matrix $\hat{L}(s)$ is used to study both the absolute and the relative stability of the system. As will be discussed in more detail in section 3, the absolute stability is assessed by applying the Generalised Nyquist Criterion (GNC) [17].

The relative stability of the system can be assessed by using the SISO gain and phase margin definitions, as discussed in [8], which requires to ignore the cross-diagonal terms of $Y_{\mathrm{c}}(s)$. An alternative stability margin, which includes such coupling terms, is presented in the following section 2.2.

\subsection{Perturbation norm stability margin}

The presented stability margin is based on the Strictly Diagonal Dominant (SDD) property of the $I+\hat{L}(s)$ over the Nyquist contour $\mathcal{D}$. This property is verified when, $\forall s \in \mathcal{D}$ [15]

$$
\begin{aligned}
& \left|1+\hat{L}_{1,1}(s)\right|>\left|\hat{L}_{1,2}(s)\right| \\
& \left|1+\hat{L}_{2,2}(s)\right|>\left|\hat{L}_{2,1}(s)\right|
\end{aligned}
$$

The results of the study presented in [16] are applied. When $I+\hat{L}(s)$ is SDD, i.e. (7) are true, then the following strictly positive quantity $d_{\infty}$ can be calculated as

$$
d_{\infty}=\min _{i=1,2 ; j \neq i}\left(\left|1+\hat{L}_{\mathrm{i}, \mathrm{i}}(s)\right|-\left|\hat{L}_{\mathrm{i}, \mathrm{j}}(s)\right|\right)
$$

with $s \in \mathcal{D}$. As demonstrated in [16], such positive value of $d_{\infty}$ is interpreted as the maximum multiplicative perturbation $\Delta L(s)$ the system can stand without losing the closed-loop stability (see Figure 3 ). For this reason, $d_{\infty}$ is named perturbation norm stability margin. By drawing the Gershgorin discs [20] of $I+\hat{L}(s)$ in the complex plane, which correspond to the regions where the eigenvalues of $I+\hat{L}(s)$ lie, the circle centred on the $(0,0)$ point and having a radius equal to $d_{\infty}$ can be identified. This is tangent to the Gershgorin discs and is called the perturbation norm circle. The quantity $d_{\infty}$ therefore represents the minimum (normed) distance between the $(0,0)$ point and the mentioned discs. A similar graphical interpretation of $d_{\infty}$ can be found in [21]. It is worth mentioning that if the matrix $I+\hat{L}(s)$ is not SDD the resulting value of $d_{\infty}$ would be negative, indicating that it is not possible to draw the perturbation norm circle. As will been discussed in section 3 , negative values of $d_{\infty}$ indicate that the stability performance of the system is likely to be poor, with less damped dynamics.

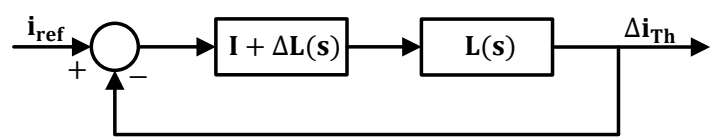

Fig. 3: Feedback loop system with applied multiplicative perturbation $\Delta L(s)$.

\subsection{Relation between SISO gain and phase margins and $d_{\infty}$}

The introduced $d_{\infty}$ margin can be related to the SISO gain and phase margins, calculated by application of the impedance-based stability criterion [8]. These will respectively be indicated as $G M_{S I S O}^{+}$and $P M_{S I S O}^{+}$ for the positive sequence and $G M_{S I S O}^{-}$and $P M_{S I S O}^{-}$for the negative sequence. 


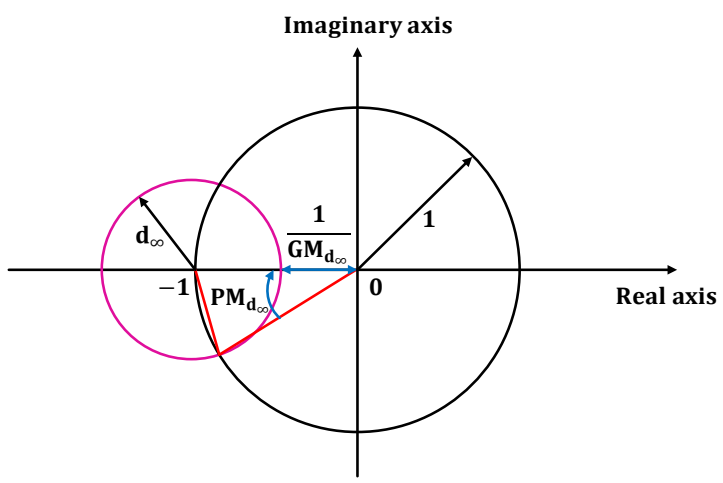

Fig. 4: Graphical equivalence between $d_{\infty}$ and SISO gain and phase margins, GM and PM respectively.

As shown in Figure 4, by definition of SISO phase margin [17], the intersection between the perturbation norm circle and the unit circle allows to calculate the equivalent minimum SISO phase margin $G M_{d_{\infty}}$ of the system, associated to $d_{\infty}$. Similarly, by definition of SISO gain margin [17], the intersection between the perturbation norm circle and the negative real axis allows to obtain the equivalent minimum SISO gain margin of the system, $G M_{d_{\infty}}$, associated to $d_{\infty}$. These minimum quantities can be calculated as

$$
\begin{aligned}
G M_{d_{\infty}}[d B] & =20 \log _{10}\left(\frac{1}{1-\min \left\{1, d_{\infty}\right\}}\right) \\
P M_{d_{\infty}}[d e g] & =\frac{360}{\pi} \arcsin \left(\frac{\min \left\{2, d_{\infty}\right\}}{2}\right)
\end{aligned}
$$

Therefore, the value of $d_{\infty}$ allows a derivation of the minimum equivalent SISO stability margins of the system. It is highlighted that (9) can be applied only if the system is SDD, i.e. $d_{\infty}>0$.

\section{Case study: relative stability of a grid-connected VSC}

In order to illustrate how the stability margin (8) can be used to assess the stability robustness of a converter-grid interface, a $1.5 \mathrm{~kW}$ laboratory prototype of a VSC connected to a grid with a low Short Circuit Ratio (SCR) is considered (see Figure 1). Its control scheme is shown in Figure 5. This has been implemented in the $q d$-frame and consists of the positive sequence current controller, and of a PLL loop to synchronise the controller operation to the grid [22].

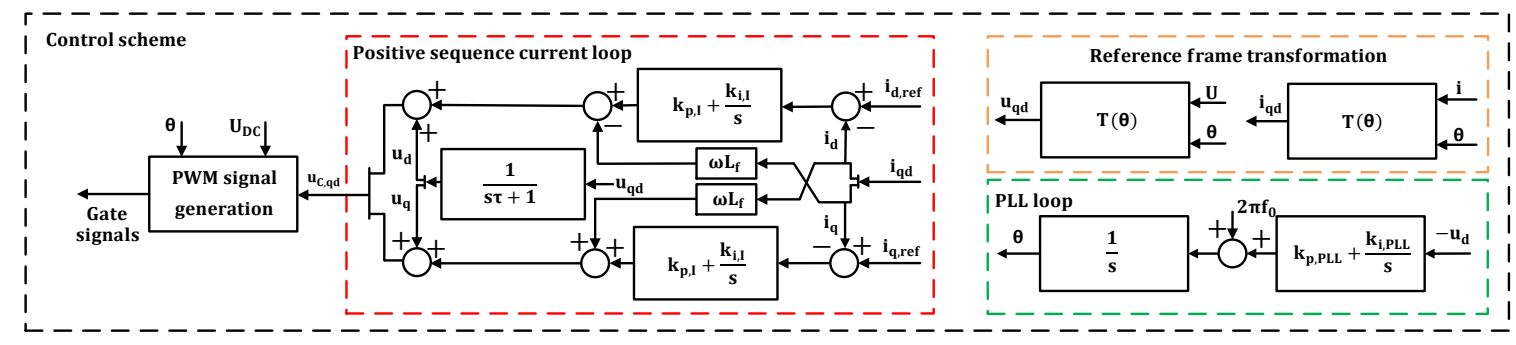

Fig. 5: Scheme of the controller employed in the carried out study.

The following Park transformation $T(t)$ has been used to refer the $a b c$-frame signals to the $q d$-frame [22]:

$$
T(t)=\frac{2}{3}\left[\begin{array}{lll}
\cos \left(\omega_{0} t\right) & \cos \left(\omega_{0} t-\frac{2}{3} \pi\right) & \cos \left(\omega_{0} t-\frac{4}{3} \pi\right) \\
\sin \left(\omega_{0} t\right) & \sin \left(\omega_{0} t-\frac{2}{3} \pi\right) & \sin \left(\omega_{0} t-\frac{4}{3} \pi\right)
\end{array}\right]
$$

The $q$-axis is aligned to phase $a$ of the measured plant voltage and leads the $d$-axis by $90^{\circ}$. The system and the control parameters are listed in Table I. 
Table I: System electrical and control parameters.

\begin{tabular}{ll}
\hline Electrical Parameters & Value \\
\hline Converter rated Power $P$ & $1.5 \mathrm{~kW}$ \\
Converter switching frequency $f_{\mathrm{s}}$ & $2.5 \mathrm{kHz}$ \\
Grid line voltage $U_{\mathrm{g}}$ & $135 \mathrm{~V}_{\text {rms }}$ \\
Grid nominal frequency & $50 \mathrm{~Hz}$ \\
Coupling inductance $L_{\mathrm{f}}$ & $2.5 \mathrm{mH}$ \\
Grid inductance $L_{\mathrm{g}}$ & $16 \mathrm{mH}$ \\
Grid resistance $R_{\mathrm{g}}$ & $1.5 \Omega$ \\
Filter capacitance $C$ & $25 \mu \mathrm{F}$ \\
Filter resistance $R$ & $33 \Omega$ \\
Inverter DC voltage & $300 \mathrm{~V}$ \\
Grid Short Circuit Ratio & 2.3 \\
\hline Controller Parameters & Value \\
\hline Current loop PI proportional gain $k_{\mathrm{I}, \mathrm{p}}$ & $1.625 \mathrm{VA}^{-1}$ \\
Current loop PI integral gain $k_{\mathrm{I}, \mathrm{i}}$ & $\left.1056.3 \mathrm{~V}^{-1} \mathrm{~A}^{-1} \mathrm{~s}^{-1}\right)$ \\
PLL loop PI proportional gain $k_{\mathrm{PLL}, \mathrm{p}}$ & $0.13 \mathrm{radV}^{-1} \mathrm{~s}^{-1}$ \\
PLL loop PI integral gain $k_{\mathrm{PLL}, \mathrm{i}}$ & $11.6 \mathrm{radV}^{-1} \mathrm{~s}^{-2}$ \\
Time constant $\tau$ & $0.1 \mathrm{~s}$ \\
\hline
\end{tabular}

In the following section 3.1, the study of the absolute and relative stability of such converter system is discussed. Thereafter, in section 3.2, an experimental validation of the analytical results is presented.

\subsection{Stability analysis}

In the carried out stability assessment, four operating points of the system in Figure 1 have been considered. These are defined by the set-points values of the $q$ - and $d$-axis current loops, $i_{\mathrm{q} \text {,ref }}$ and $i_{\mathrm{d} \text {,ref }}$ respectively, as detailed in Table II.

Table II: Current loop set-points for the considered operating points of the system.

\begin{tabular}{lll}
\hline Operating point & $i_{\mathbf{q}, \text { ref }}$ & $i_{\mathbf{d}, \text { ref }}$ \\
\hline$O P_{1}$ & $3 \mathrm{~A}$ & $0 \mathrm{~A}$ \\
$O P_{2}$ & $4 \mathrm{~A}$ & $0 \mathrm{~A}$ \\
$O P_{3}$ & $5 \mathrm{~A}$ & $0 \mathrm{~A}$ \\
$O P_{4}$ & $6 \mathrm{~A}$ & $0 \mathrm{~A}$ \\
\hline
\end{tabular}

Figures $6 \mathrm{a}$ and $6 \mathrm{~b}$ show the results of the verification of the conditions in (7), for the first and the second rows of the $I+\hat{L}(s)$ matrix, respectively. As it can be seen, the system is SDD for all the tested operating points, but for $\mathrm{OP}_{4}$. As such, according to the theory illustrated in section 2.2, the resulting perturbation norm stability margin $d_{\infty}$ is positive only for $O P_{1}, O P_{2}$ and $O P_{3}$.

The stability of the system can be assessed by applying the Generalised Nyquist Criterion (GNC) to $I+$ $L(s)$ [17], which is equivalent to applying the Generalised Inverse Nyquist Criterion (GINC) to $I+\hat{L}(s)$ [23], [24]. The latter criterion has been used in this work. As $\hat{L}(s)$ is open-loop stable [24], in order to verify the absolute stability of the system, it is enough to verify that the characteristic loci of $I+\hat{L}(s)$ do not encircle the $(0,0)$ point.

The Nyquist plots of $I+\hat{L}(s)$ are shown in Figure 7 for the different considered operating points. The associated Gershgorin discs are also shown. For the operating points $O P_{1}, O P_{2}$ and $O P_{3}$ it has been possible to draw the perturbation norm circle, thanks to the SDD property of the system, which makes $d_{\infty}>0$. Contrarily, as for $\mathrm{OP}_{4}$ such property is not verified, the resulting Gershgorin discs encircle the $(0,0)$ point (see Figure $7 \mathrm{~d}$ ), and $d_{\infty}$ is negative.

The observed reduction in the size of the perturbation circle (see Figures 7a, 7b, and 7c) indicates that, despite of the fact that the system is absolute stable, its relative stability decreases as $i_{\mathrm{q}, \text { ref }}$ increases.

For the calculated $d_{\infty}$ positive values, the corresponding equivalent minimum SISO margins have been derived, based on (9). These have been compared to the SISO stability margins obtained by application of the impedance based stability-criterion [8], i.e. neglecting the off-diagonal terms of $\hat{L}(s)$. The Bode plots 


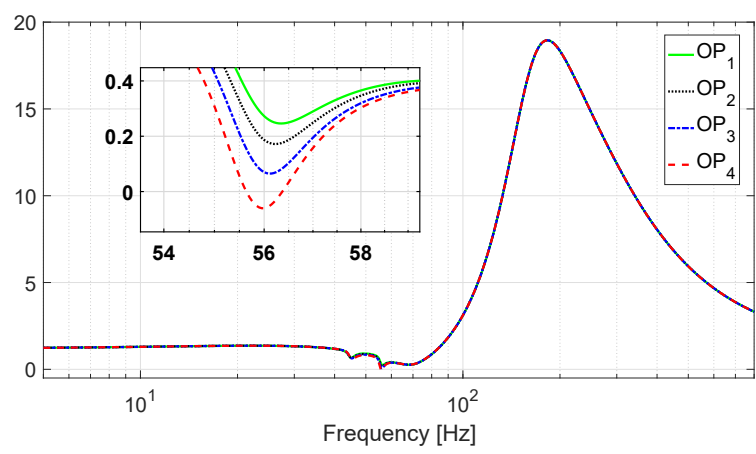

(a)

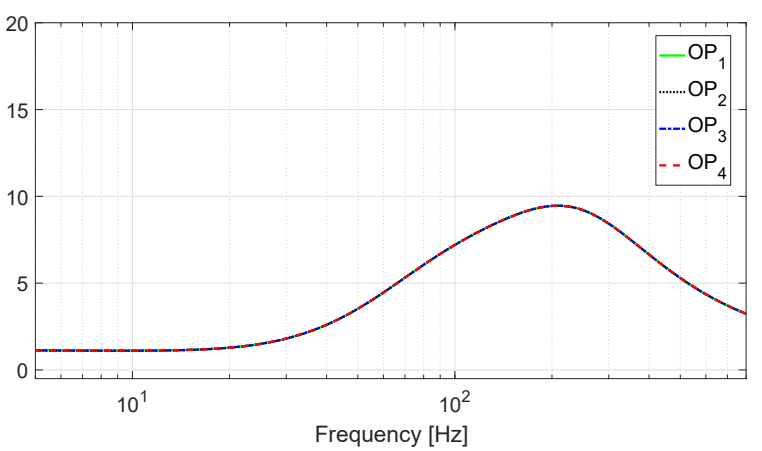

(b)

Fig. 6: Verification of the diagonal dominance property of the system for the considered operating points; (a) $\left|1+\hat{L}_{1,1}(j 2 \pi f)\right|-\left|\hat{L}_{1,2}(j 2 \pi f)\right|$; (b) $\left|1+\hat{L}_{2,2}(j 2 \pi f)\right|-\left|\hat{L}_{2,1}(j 2 \pi f)\right|$.

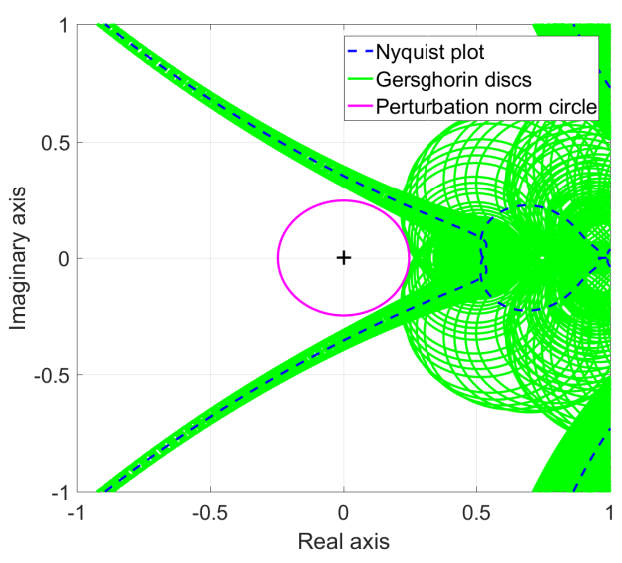

(a)

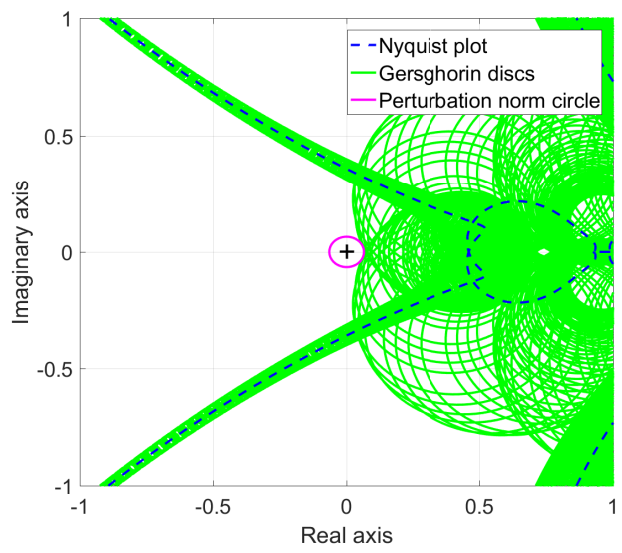

(c)

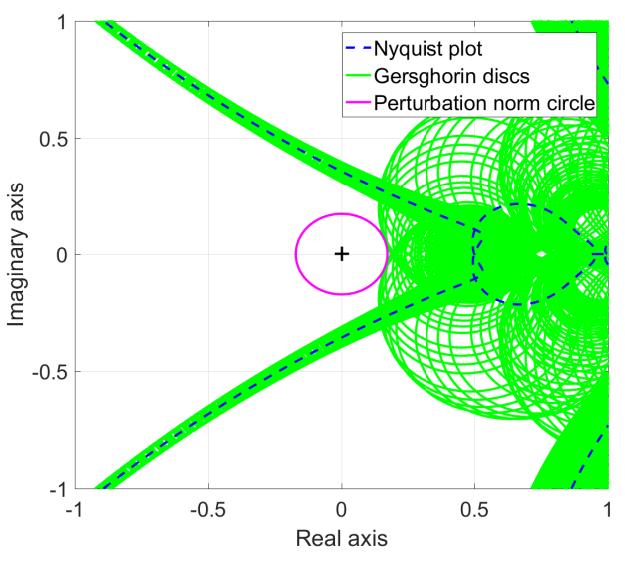

(b)

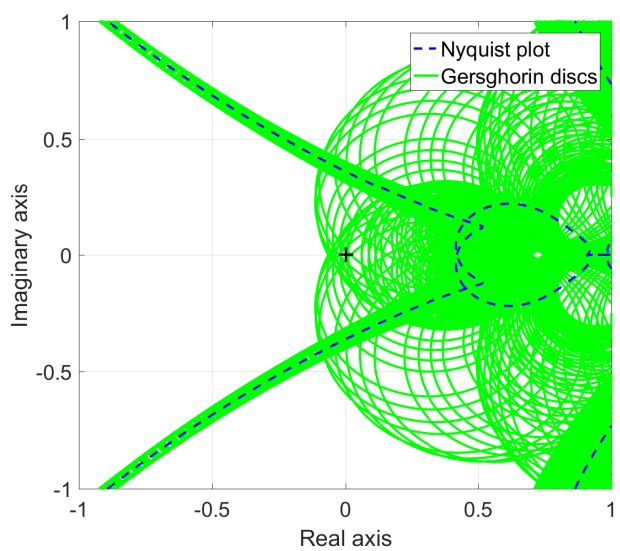

(d)

Fig. 7: Nyquist plots of $I+\hat{L}(s)$ for the considered operating points: (a) $O P_{1}$, (b) $O P_{2}$, (c) $O P_{3}$, (d) $O P_{4}$. The Gershgorin discs are also shown, together with the perturbation norm circle for the scenarios where the system is SDD. 

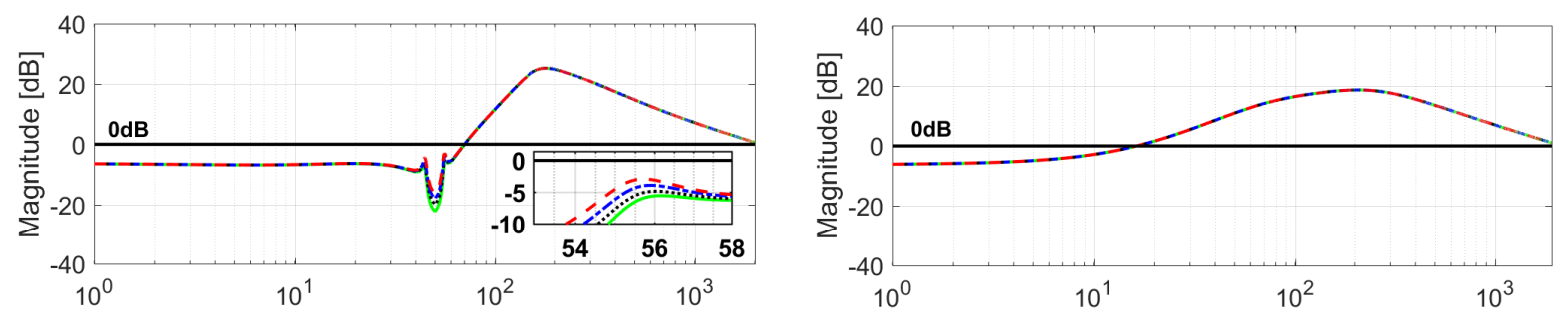

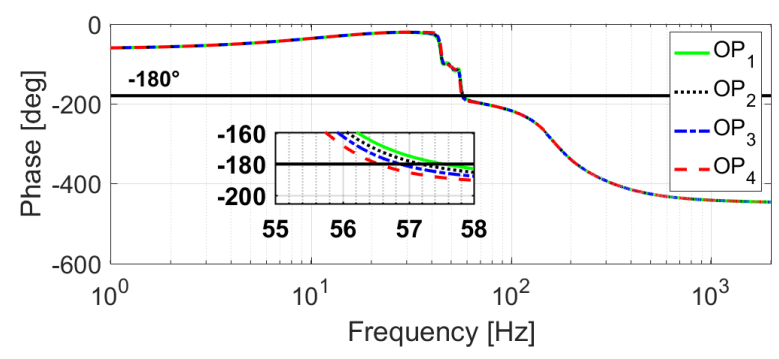

(a)

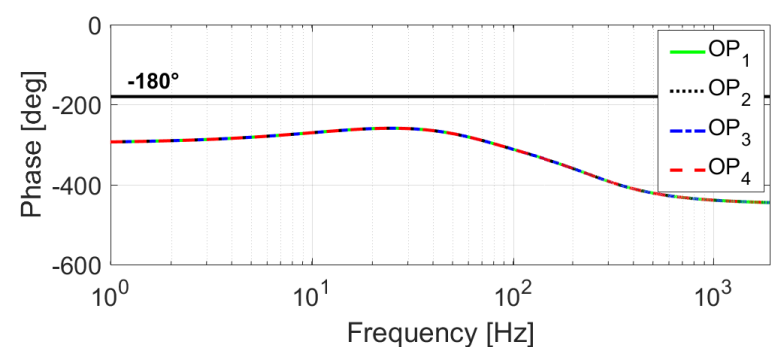

(b)

Fig. 8: Bode plots of $\hat{L}_{1,1}(j 2 \pi f)$ (a) and $\hat{L}_{2,2}(j 2 \pi f)$ (b).

of $\hat{L}_{1,1}(j 2 \pi f)$ and $\hat{L}_{2,2}(j 2 \pi f)$ are shown in Figure 8, from which the aforementioned stability margins $G M_{S I S O}^{+}, P M_{S I S O}^{+}, G M_{S I S O}^{-}$and $P M_{\text {SISO }}^{-}$have been calculated. Table III compares the obtained stability margins figures. These data indicate that the critical resonance frequency of the system is around $57 \mathrm{~Hz}$. As it can be seen, the SISO gain margin $G M_{\text {SISO }}^{+}$decreases as $i_{\text {q,ref }}$ increases, indicating a poorer relative stability of the system at higher current generation levels. This is also confirmed by the corresponding reduction in $d_{\infty}$, and then by the loss of the system SDD property for $O P_{4}$, which makes $d_{\infty}$ negative.

The SISO figures associated to the calculated positive $d_{\infty}$ margin indicate its conservative feature. Such conservativeness relies on the significance of $d_{\infty}$ [16]. As aforementioned, when the system is SDD, $d_{\infty}$ is the upper limit on the perturbation $\Delta L(s)$ that can be applied to the system without making it unstable. In general, $\Delta L(s)$ is not diagonal, as such it can affect both the positive and negative sequence control loops simultaneously. Because $d_{\infty}$ is calculated taking into account the off-diagonal terms of $\hat{L}(s)$ (see (8)), and any generic perturbation applied on the system is considered (i.e. also a non diagonal perturbation), such stability margin results in a more conservative index. By taking the coupling between the positive and negative sequence loops into account, a safer measure of the system relative stability is therefore obtained.

Table III: Comparison between the SISO stability margins calculated with the impedance-based stability criterion $\left(G M_{S I S O}^{+}, F M_{S I S O}^{+}, G M_{S I S O}^{-}, P M_{S I S O}^{-}\right)$and the alternative figures based on the study of the system diagonal dominance ( $d_{\infty}$ and its equivalent SISO quantities $G M_{d_{\infty}}$ and $P M_{d_{\infty}}$ ).

\begin{tabular}{llllllll}
\hline$O P$ & $G M_{S I S O}^{+}[\mathbf{d B}]$ & $P M_{S I S O}^{+}[\mathbf{d e g}]$ & $G M_{S I S O}^{-}[\mathbf{d B}]$ & $G M_{S I S O}^{-}[\mathbf{d e g}]$ & $d_{\infty}$ & $G M_{d_{\infty}}[\mathbf{d B}]$ & $P M_{d_{\infty}}[\mathrm{deg}]$ \\
\hline$O P_{1}$ & $6.2($ at $57.5 \mathrm{~Hz})$ & $19($ at $70.2 \mathrm{~Hz})$ & $\infty$ & $83($ at $16 \mathrm{~Hz})$ & 0.25 & $\geq 2.5$ & $\geq 14.4$ \\
$O P_{2}$ & $5.7($ at $57.2 \mathrm{~Hz})$ & $19($ at $70.1 \mathrm{~Hz})$ & $\infty$ & $83($ at $16 \mathrm{~Hz})$ & 0.17 & $\geq 1.6$ & $\geq 9.8$ \\
$O P_{3}$ & $4.8($ at $56.9 \mathrm{~Hz})$ & $19($ at $70.1 \mathrm{~Hz})$ & $\infty$ & $83($ at $16 \mathrm{~Hz})$ & 0.07 & $\geq 0.6$ & $\geq 4$ \\
$O P_{4}$ & $3.9($ at $56.6 \mathrm{~Hz})$ & $19($ at $70 \mathrm{~Hz})$ & $\infty$ & $83($ at $16 \mathrm{~Hz})$ & -0.06 & - & - \\
\hline
\end{tabular}

\subsection{Experimental validation}

The analytical results presented in section 3.1 have been verified experimentally making use of the laboratory prototype of the studied system, whose picture is shown in Figure 9.

The system has been tested for a staircase-like increase of the $i_{\mathrm{q}, \text { ref }}$ set-point from $3 \mathrm{~A}$ up to $7 \mathrm{~A}$. The recorded data are shown in Figure 10a. A spectral analysis of each of the four step-response transients occurring during the $\Delta T_{1}, \Delta T_{2}, \Delta T_{3}$ and $\Delta T_{4}$ periods highlighted in Figure 10a is shown in Figure 10b. As it can be seen, the transients present a spectral peak at $\approx 6 \mathrm{~Hz}$ in the $q d$-frame, which corresponds to a 
positive-sequence mode at $\approx 56 \mathrm{~Hz}$ in the $a b c$-frame. The magnitude of this spectral peak increases with $i_{\mathrm{q}, \mathrm{ref}}$, indicating less damping of the system dynamics at higher current generation levels. The presence of smaller spectral peaks at $\approx 106 \mathrm{~Hz}$, which indicates the existence of a negative-sequence mode at $\approx 56 \mathrm{~Hz}$ in the $a b c$-frame, is attributed to minor imbalances in the electrical impedances.

These data confirm the conclusions of the presented analytical study, providing a substantial overlap between the critical resonance frequency predicted theoretically $(\approx 57 \mathrm{~Hz})$ and the one verified experimentally $(\approx 56 \mathrm{~Hz})$.

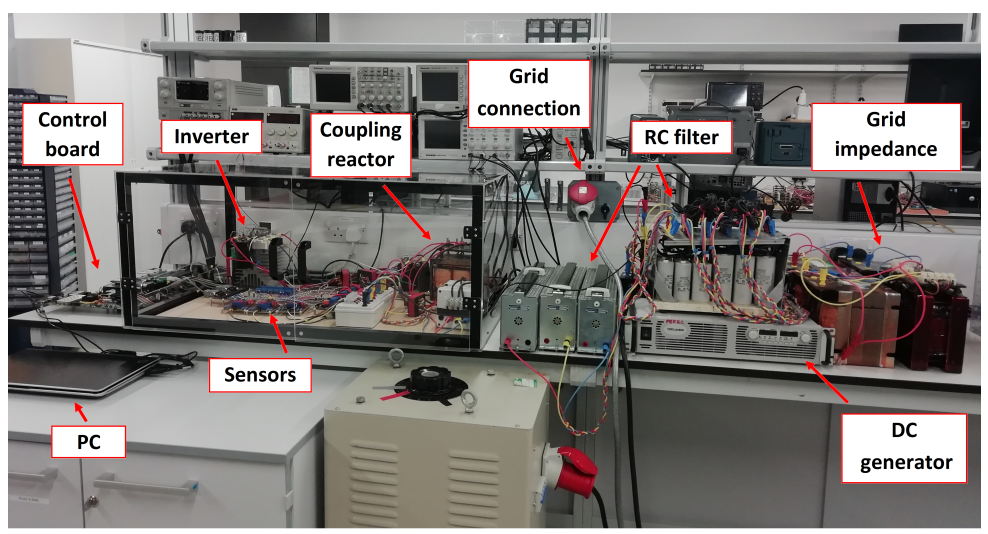

Fig. 9: Picture of the built grid-connected VSC prototype.

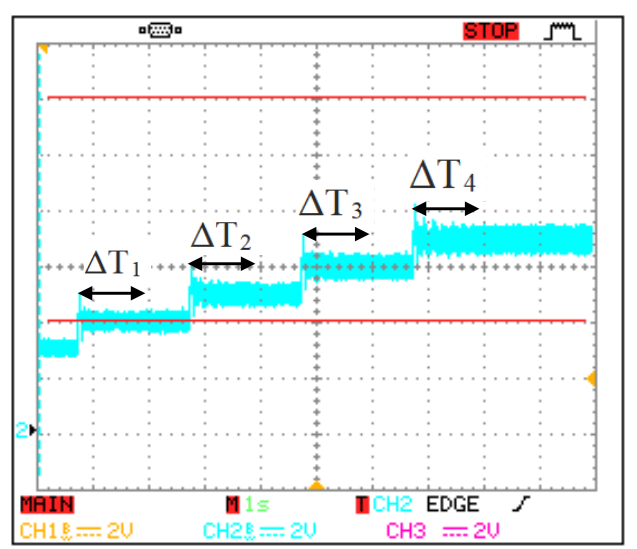

(a)

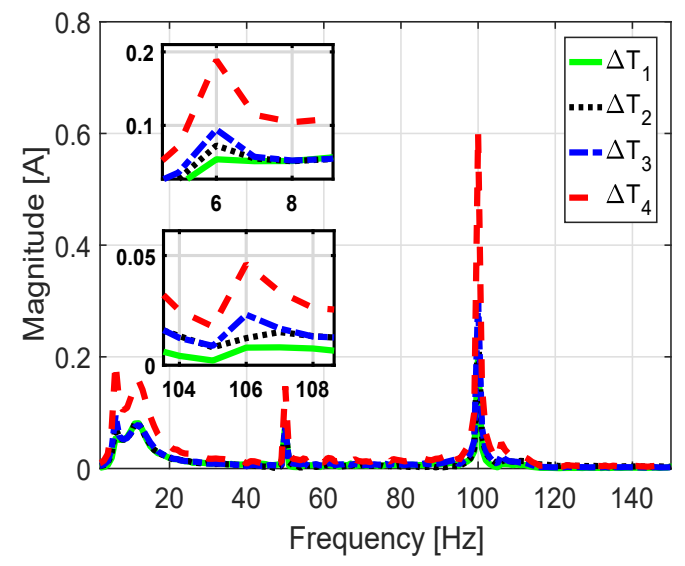

(b)

Fig. 10: (a) Recorded response of $i_{\mathrm{q}}(t)$ for a staircase-like increase of $i_{\mathrm{q}, \text { ref }}(t)$ from $3 \mathrm{~A}$ to $7 \mathrm{~A}$. (b) Magnitude spectra of the recorded transients, occurring during the $\Delta T_{1}, \Delta T_{2}, \Delta T_{3}$ and $\Delta T_{4}$ periods.

\section{Conclusion}

A perturbation norm stability margin has been proposed, which can be used for the stability study of a converter-grid interface. This stability margin is based on the property of diagonal dominance of such converter system when it is represented in terms of small-signal impedances in the sequence-frame. As its calculation takes into account the coupling between the positive and negative sequence small-signal converter admittance terms, a safer and more conservative stability index can be obtained compared to the conventional SISO stability margins used in the impedance-based stability criterion. The presented theory has been applied to assess how the stability robustness of a VSC system connected to a weak grid varies with its operating point. The poorer damping of the system dynamics, which occurs at higher current generation levels, has been correctly indicated by the proposed stability margin, thus proving its effectiveness. Experimental results with a real converter-prototype have been used to support the analytical study. 


\section{References}

[1] Lawrence J.E.: Renewable energy integration: practical management of variability, uncertainty, and flexibility in power grids, Academic Press 2017

[2] Zhu J. and Booth C.: Future multi-terminal HVDC transmission systems using voltage source converters, IEEE Universities Power Engineering Conference (UPEC), 2010 45th International pp 1- 6

[3] Brogan P.: The stability of multiple, high power, active front end voltage sourced converters when connected to wind farm collector systems, 2010 Proc. EPE Wind Energy Chapter Seminar

[4] Leon A. E and Solsona J.A.: Sub-synchronous interaction damping control for DFIG wind turbines, IEEE Transactions on Power Systems Vol. 30, no. 1, pp. 419- 428, 2015

[5] Egea-Àlvarez A. and Fekriasl S. and Hassan F. and Gomis-Bellmunt O.: Advanced vector control for voltage source converters connected to weak grids, IEEE Transactions on Power Systems Vol. 30 no. 6, pp. 30723081, 2015

[6] Blaabjerg F. and Teodorescu R. and Liserre M. and Timbus, A.V.: Overview of control and grid synchronization for distributed power generation systems, IEEE Transactions on industrial electronics Vol. 53, no. 5, pp. 1398- 1409, 2006

[7] Sainz L. and Cheah-Mane M. and Monjo L. and Liang J. and Gomis-Bellmunt O.: Positive-Net-Damping Stability Criterion in Grid-Connected VSC Systems, IEEE Journal of Emerging and Selected Topics in Power Electronics Vol. 5, no. 4, pp. 1499- 1512, 2017

[8] Sun J.: Impedance-based stability criterion for grid-connected inverters, IEEE Transactions on Power Electronics Vol. 26 no. 11, pp. 3075- 3078, 2011

[9] Rygg A. and Molinas M. and Zhang C. and Cai X.: A Modified Sequence-Domain Impedance Definition and Its Equivalence to the dq-Domain Impedance Definition for the Stability Analysis of AC Power Electronic Systems, Journal of Emerging and Selected Topics In Power Electronics Vol. 4 no. 4, pp. 1383- 1396, 2016

[10] Safonov M. and Athans M.: A multiloop generalization of the circle criterion for stability margin analysis, IEEE Transactions on Automatic Control Vol. 26, no. 2, pp. 415- 422, 1981

[11] Wang X. and Harnefors L. and Blaabjerg F. and Loh P.C.: A unified impedance model of voltage-source converters with phase-locked loop effect, Energy Conversion Congress and Exposition (ECCE), 2016 IEEE, pp. $1-8,2016$

[12] Wang X. and Harnefors L. and Blaabjerg F.: Unified Impedance Model of Grid-Connected Voltage-Source Converters, IEEE Transactions on Power Electronics Vol. 33 no. 2, pp. 1775- 1787, 2018

[13] Latchman HA. and Crisalle OD.: Exact robustness analysis for highly structured frequency-domain uncertainties, American Control Conference, Proceedings of the 1995 Vol. 6, pp. 3982- 3987, 1995

[14] Doyle J.: Analysis of feedback systems with structured uncertainties, IEE Proceedings D-Control Theory and Applications Vol. 129, no. 6, pp. 242- 250, 1982

[15] Johnson M.A.: Diagonal dominance and the method of pseudodiagonalisation, IET Proceedings of the Institution of Electrical Engineers Vol. 126 no. 10, pp. 1011- 1017, 1979

[16] Yeung L.F. and Bryant G.F.: Robust stability of diagonally dominant systems, IEE Proceedings D (Control Theory and Applications) Vol. 131 no. 6, pp. 253- 260, 1984

[17] Doyle J.C. and Francis B.A. and Tannenbaum A.R.: Feedback control theory, Courier Corporation, 2013

[18] Rygg A. and Molinas M. and Zhang C. and Cai X.: On the Equivalence and Impact on Stability of Impedance Modeling of Power Electronic Converters in Different Domains, IEEE Journal of Emerging and Selected Topics in Power Electronics Vol. 5 no. 4, pp. 1444- 1454, 2017

[19] Kirtley J. L.: Electric power principles: sources, conversion, distribution and use, John Wiley \& Sons, 2011

[20] Juang Y.T. and Shao C.S.: Stability analysis of dynamic interval systems, International Journal of Control Vol. 49 no. 4, pp. 1401- 1408, 1989

[21] Owens D.H. and Chotai A: Robust controller design for linear dynamic systems using approximate models, IEE Proceedings D (Control Theory and Applications) Vol. 130 no. 2, pp. 45- 56, 1983

[22] Yazdani A. and Iravani R.: Voltage-sourced converters in power systems: modeling, control, and applications, John Wiley \& Sons, 2010

[23] Taylor and Francis: A generalized inverse Nyquist stability criterion, International Journal of Control Vol. 23, no. 3, pp. 325- 340, 1977

[24] Wen B. and Boroyevich D. and Burgos R. and Mattavelli, P. and Shen, Z.: Inverse Nyquist stability criterion for grid-tied inverters, IEEE Transactions on Power Electronics Vol. 32, no. 2, pp. 1548- 1556, 2017 